\title{
Understanding the pattern of lymph node metastasis for trans- segmental thoracic esophageal cancer to develop precise delineation of clinical target volume for radiotherapy
}

\author{
Liyuan Huang, Chen Chen, Mingqiang Lin, Jiancheng Li \\ Department of Radiation Oncology, Fujian Cancer Hospital \& Fujian Medical University Cancer Hospital, Fujian 350014, China \\ Contributions: (I) Conception and design: L Huang, J Li; (II) Administrative support: J Li; (III) Provision of study materials or patients: J Li; (IV) \\ Collection and assembly of data: L Huang, C Chen, M Lin; (V) Data analysis and interpretation: L Huang; (VI) Manuscript writing: All authors; (VII) \\ Final approval of manuscript: All authors. \\ Correspondence to: Jiancheng Li. Department of Radiation Oncology, Fujian Cancer Hospital \& Fujian Medical University Cancer Hospital, No. 420, \\ Fumalu Road, Jinan District, Fuzhou 350014, China. Email: jianchengli6@163.com.
}

\begin{abstract}
Background: This study was conducted to explore the lymph node metastasis (LNM) pattern of thoracic esophageal cancer (TEC) depending upon the location of the primary tumor and provide a reference for the design of clinical target volume (CTV).

Methods: The data of patients who underwent radical esophagectomy and three-field lymph node dissection at Fujian Cancer Hospital from 2006 to 2010 were retrospectively analyzed. We segmented the esophagus according to the anatomical landmarks on computed tomography (CT) and defined the transsegmental and mono-segmental esophageal carcinoma. The LNM pattern in trans-segmental and monosegmental esophageal cancer was explored and the CTV delineation was determined based on LNM pattern. Results: A total of 852 patients were included in this study. The top five sites of LNM for upper-middle TEC were cervical, upper and middle paraesophageal, and zone 1, 2, 4 regions. The most common sites of LNM for lower-middle TEC were cervical and middle paraesophageal, group 3, 7, and zone 7 regions. The top five sites of LNM for middle-upper TEC were cervical, middle paraesophageal, zone 1, 7, and group 7 regions. The most common sites of LNM for middle-lower TEC were cervical, middle paraesophageal, zone 7, and group 2, 7 regions. The top five sites of LNM for TEC involving all the segments were cervical, middle paraesophageal, zone 7 , group 2 and 7 regions.

Conclusions: LNM pattern of trans-segmental and mono-segmental TEC varies depending upon the primary tumor location. The irradiation field must be designed according to the primary tumor location.
\end{abstract}

Keywords: Esophageal cancer (EC); lymph node metastasis (LNM); clinical target volume (CTV)

Submitted Sep 18, 2019. Accepted for publication Feb 11, 2020.

doi: 10.21037/apm.2020.04.10

View this article at: http://dx.doi.org/10.21037/apm.2020.04.10

\section{Introduction}

Esophageal cancer (EC) is a common upper digestive tract malignant tumor especially in eastern Asia and Africa. It is the seventh most common cause of morbidity and sixth most common cause of mortality among the malignant tumors worldwide (1). Despite advances in the multimodality treatment of EC, the 5-year survival rate of esophageal cancer continues to remain low (-40\%) (2). Lymph node metastasis $(\mathrm{LNM})$ is one of the most significant prognostic factors affecting the survival of EC (3).

The Union for International Cancer Control (UICC) divided the esophagus into four segments. Different tumor locations have different patterns of LNM, which plays an important role in the evaluation and treatment of EC. Usually, radiation oncologists delineate clinical 
target volume (CTV) focusing on areas with high rates of LNM based on the location of tumor, which still remains disputed. Moreover, thoracic esophageal cancer (TEC) spanning segments are often encountered, whose LNM characteristics can be different from mono-segmental TEC. But the principles of TEC spanning are poorly understood. The benefits of precision radiotherapy may be offset by following the same criteria of CTV delineation. This retrospective study aimed to investigate the LNM pattern of trans-segmental TEC which can help in designing CTV.

\section{Methods}

This retrospective study was approved by Fujian Cancer Hospital Institutional Review Board. All patients provided written informed consent prior to treatment, and all information was anonymized prior to analysis.

In this study, we included patients with TEC who underwent surgery during the study period from January 2006 to December 2010 at Fujian Cancer Hospital. The patients who satisfied the following conditions were included: (I) histologically proven primary esophageal squamous cell cancer, (II) the upper and lower boundaries of the tumor were clearly defined by endoscopy, esophageal barium meal, and high-resolution CT chest examination before operation in our hospital, and (III) underwent radical esophagectomy and three-field lymph node dissection. The exclusion criteria were: patients who received preoperative radiotherapy or chemotherapy, those having cervical esophageal cancer and those with distant metastasis. Patients with prior thoracic surgery before esophagectomy were also excluded.

According to the 8th edition of the American Joint Committee on Cancer (AJCC), the location of esophageal tumor was defined based on the center of the tumor. In the present study, the location of the tumor was determined using the anatomical landmarks on preoperative highresolution CT examination. The term 'upper/middle/ lower thoracic esophageal cancer' (U/M/L TEC) were specifically used for the tumors whose boundaries were in the same segment. For larger tumors located in more than one segment, we used the term 'trans-segmental esophageal carcinoma'. The upper-middle thoracic esophageal cancer (U-M TEC) referred to the tumor whose center was within upper esophagus while the lower border extended in to the middle esophagus. Similarly, the middle-upper thoracic esophageal cancer (M-U TEC) referred to the tumor whose center was in the middle esophageal segment while the upper border was extending in to the upper esophagus. The middle-lower thoracic esophageal cancer (M-L TEC) referred to the tumor whose center was within middle esophagus while the lower border was extending in to the lower esophageal segment. The lower-middle thoracic esophageal cancer (L-M TEC) refers to the tumor whose center was within the lower esophagus while the upper boundary extended in to the middle esophagus. The tumors extending to all the three segments of the thoracic esophagus were termed as 'all thoracic esophageal cancer' (ATEC).

To accurately describe the pattern of LNM, the regional lymph nodes were named as described by the Japanese Society for Esophageal Diseases (JSED) and AJCC. The characteristics of LNM pattern were collected retrospectively from postoperative pathological LN status. Then we compared the differences in LNM between transsegmental and mono-segmental TEC, to make precise delineation of CTV for routine clinical practice.

Categorical data are presented as numbers and percentages. The LNM rates were compared between the groups by Chi-square test or Fisher's exact test as applicable. Data were analyzed using Statistical Package for Social Sciences (SPSS, Chicago, IL, USA) version 22.0 for Windows. A two-tailed $\mathrm{P}$ value $<0.05$ was considered statistically significant.

\section{Results}

A total of 852 patients with TEC were included in this study. The median age was 57 years (range, 31 to 84 years). There were 649 males (76.2\%) and 203 females (23.8\%). The numbers of patients with $\mathrm{U}, \mathrm{U}-\mathrm{M}, \mathrm{M}, \mathrm{M}-\mathrm{U}, \mathrm{M}-\mathrm{L}$, A, L, and L-M TEC were 88 (10.3\%), 44 (5.2\%), 285 (33.5\%), 150 (17.6\%), 198 (23.3\%), 25 (2.9\%), 48 (5.6\%), and $14(1.6 \%)$, respectively. The demographic details of the patients are shown in Table 1.

The incidence of LNM varied depending upon the location of the primary tumor. Even for the TEC of the same segment of esophagus, the LNM pattern of transsegmental and mono-segmental TEC were different. The rates of LNM in neck, upper mediastinum, middle mediastinum, lower mediastinum, and abdominal cavity were $36.8 \%$ (313/852), 8.3\% (71/852), 23.5\% (200/852), $9.4 \%$ (80/852), and 23.4\% (199/852), respectively (Table 2). The LNM rates of patients with U TEC in neck, upper mediastinum, middle mediastinum, lower mediastinum, and abdominal cavity were $47.7 \%(42 / 88), 5.7 \%(5 / 88)$, $8.0 \%(7 / 88), 2.3 \%(2 / 88)$, and $2.3 \%$ (2/88), respectively. 
Table 1 The clinical characteristics of 852 patients with TEC

\begin{tabular}{|c|c|c|c|c|c|c|c|c|c|}
\hline Characteristics & $U$ & U-M & $M$ & $M-U$ & $M-L$ & $A$ & $\mathrm{~L}$ & L-M & Total \\
\hline \multicolumn{10}{|l|}{ Sex } \\
\hline Male & 60 & 36 & 213 & 119 & 150 & 23 & 37 & 11 & 649 \\
\hline Female & 28 & 8 & 72 & 31 & 48 & 2 & 11 & 3 & 203 \\
\hline Tis & 1 & 0 & 4 & 0 & 0 & 0 & 0 & 0 & 5 \\
\hline $\mathrm{T} 1$ & 16 & 0 & 52 & 10 & 3 & 1 & 11 & 0 & 94 \\
\hline $\mathrm{T} 2$ & 20 & 11 & 49 & 25 & 34 & 2 & 10 & 2 & 155 \\
\hline T3 & 31 & 26 & 135 & 89 & 125 & 15 & 21 & 11 & 446 \\
\hline \multicolumn{10}{|l|}{$\mathrm{N}$} \\
\hline NO & 39 & 17 & 135 & 49 & 66 & 7 & 16 & 5 & 334 \\
\hline $\mathrm{N} 1$ & 23 & 14 & 69 & 48 & 62 & 9 & 17 & 6 & 248 \\
\hline $\mathrm{N} 2$ & 21 & 9 & 59 & 33 & 40 & 3 & 10 & 2 & 177 \\
\hline N3 & 5 & 4 & 22 & 20 & 30 & 6 & 5 & 1 & 93 \\
\hline \multicolumn{10}{|l|}{ Differentiation } \\
\hline High & 3 & 3 & 30 & 24 & 18 & 1 & 2 & 1 & 82 \\
\hline Middle & 66 & 31 & 203 & 93 & 145 & 21 & 37 & 10 & 606 \\
\hline Low & 12 & 8 & 36 & 25 & 19 & 0 & 7 & 1 & 108 \\
\hline
\end{tabular}

TEC, thoracic esophageal cancer; U, upper thoracic esophageal cancer; U-M, upper-middle thoracic esophageal cancer; M, middle thoracic esophageal cancer; $\mathrm{M}-\mathrm{U}$, middle-upper thoracic esophageal cancer; M-L, middle-lower thoracic esophageal cancer; A, all thoracic esophageal cancer; L, lower thoracic esophageal cancer; L-M, lower-middle thoracic esophageal cancer.

Table 2 LNM rates at different sites taking all the TEC cases in to consideration

\begin{tabular}{lccccc}
\hline & Neck (\%) & Um (\%) & Mm (\%) & Lm (\%) & Ac (\%) \\
\hline TEC & $313 / 852(36.8)$ & $71 / 852(8.3)$ & $200 / 852(23.5)$ & $80 / 852(9.4)$ & $199 / 852(23.4)$ \\
\hline
\end{tabular}

LNM, lymph node metastasis; TEC, thoracic esophageal cancer; Um, upper mediastinum; Mm, middle mediastinum; Lm, lower mediastinum; Ac, abdominal cavity.

The LNM rate was $45.5 \%$ (20/44), $25.0 \%$ (11/44), $6.8 \%$ (3/44), $2.3 \%$ (1/44), and 6.8\% (3/44) for patients with U-M TEC respectively (Table 3). The LNM rates were $12.5 \%$
(6/48), $4.2 \%$ (2/48), $25.0 \%$ (12/48), 10.4\% (5/48), and $47.9 \%(23 / 48)$ in patients with L TEC and $42.9 \%(6 / 14)$, $0 \%(0 / 14), 21.4 \%(3 / 14), 8.3 \%(4 / 14)$, and $42.9 \%(6 / 14)$ in 
Table 3 LNM rates at different sites for upper (U) and upper-middle (U-M) TEC

\begin{tabular}{|c|c|c|c|c|c|c|}
\hline U/U-M TEC & Neck (\%) & Um (\%) & Mm (\%) & $\operatorname{Lm}(\%)$ & Ac (\%) & $\mathrm{n}$ \\
\hline U-M & $20(45.5)$ & $11(25.0)$ & $3(6.8)$ & 1 (2.3) & $3(6.8)$ & 44 \\
\hline$x^{2}$ & 0.061 & 10.277 & 0 & 0 & 0.650 & \\
\hline$P$ & 0.805 & 0.001 & 1 & 1 & 0.420 & \\
\hline
\end{tabular}

LNM, Iymph node metastasis; U, upper thoracic esophageal cancer; U-M, upper-middle thoracic esophageal cancer; Um, upper mediastinum; Mm, middle mediastinum; Lm, lower mediastinum; Ac, abdominal cavity.

Table 4 LNM rates at different sites for lower (L) and lower-middle (L-M) TEC

\begin{tabular}{lccccc}
\hline L/L-M TEC & Neck (\%) & Um (\%) & Mm (\%) & Lm (\%) & Ac (\%) \\
\hline L & $6(12.5)$ & $2(4.2)$ & $12(25.0)$ & $5(10.4)$ & $23(47.9)$ \\
L-M & $6(42.9)$ & $0(0)$ & $3(21.4)$ & $4(8.3)$ & $6(42.9)$ \\
$X^{2}$ & 4.602 & 0 & 0 & 1.602 & 0.111 \\
P & 0.032 & 1 & 1 & 0.206 & 0.739 \\
\hline
\end{tabular}

LNM, Iymph node metastasis; L, lower thoracic esophageal cancer; L-M, lower-middle thoracic esophageal cancer; Um, upper mediastinum; Mm, middle mediastinum; Lm, lower mediastinum; Ac, abdominal cavity.

Table 5 LNM rates to different regions for $\mathrm{M}, \mathrm{M}-\mathrm{U}, \mathrm{M}-\mathrm{L}$ and A TEC

\begin{tabular}{lcccccc}
\hline M/M-U/M-L/A TEC & Neck (\%) & Um (\%) & Mm (\%) & Lm (\%) & Ac (\%) \\
\hline M & $90(31.6)$ & $14(4.9)$ & $54(18.9)$ & $20(7.0)$ & $61(21.4)$ & 285 \\
M-U & $76(50.7)$ & $21(14.0)$ & $42(28.0)$ & $20(13.3)$ & $28(18.7)$ & 150 \\
M-L & $65(32.8)$ & $14(7.1)$ & $68(34.3)$ & $23(11.6)$ & $59(34.9)$ & 198 \\
A & $7(28.0)$ & $4(16.0)$ & $11(44.0)$ & $120.0)$ & $7(28.0)$ \\
X $^{2}$ & 17.936 & 13.349 & 0.711 & 15.504 \\
P & 0.001 & 0.004 & 0.001 & 0.052 & 0.001 \\
\hline
\end{tabular}

LNM, lymph node metastasis; M, middle thoracic esophageal cancer; M-U, middle-upper thoracic esophageal cancer; M-L, middlelower thoracic esophageal cancer; A, all thoracic esophageal cancer; Um, upper mediastinum; Mm, middle mediastinum; Lm, lower mediastinum; Ac, abdominal cavity.

patients with L-M TEC respectively (Table 4). The LNM rates in neck, upper mediastinum, middle mediastinum, lower mediastinum, and abdominal cavity were $31.6 \%$ (90/285), 4.9\% (14/285), 18.9\% (54/285), 7.0\% (20/285), and $21.4 \%(61 / 285)$, respectively in patients with $M$ TEC. The LNM rates were $50.7 \%$ (76/150), 14.0\% (21/150), $28.0 \%$ (42/150), $13.3 \%(20 / 150)$, and $18.7 \%(28 / 150)$ in patients with M-U TEC, 32.8\% (65/198), 7.1\% (14/198), $34.3 \%$ (68/198), $11.6 \%(23 / 198)$, and $34.9 \%(69 / 198)$ in patients with M-L TEC and 28.0\% (7/25), 16.0\% (4/25), $44.0 \%(11 / 25), 20.0 \%(5 / 25)$, and $28.0 \%(7 / 25)$ in patients with A TEC respectively (Table 5).

Table 6 showed the top five sites of LNM rates in TEC with different tumor location.

\section{Discussion}

$\mathrm{EC}$ is one of the commonest digestive tract cancers associated with high incidence of morbidity and mortality. In recent years, the prognosis has significantly improved with the multimodality treatment, however the 5-year survival rate is still low because of the early metastasis. 
Table 6 The top five sites of LNM rates based on the primary tumor location

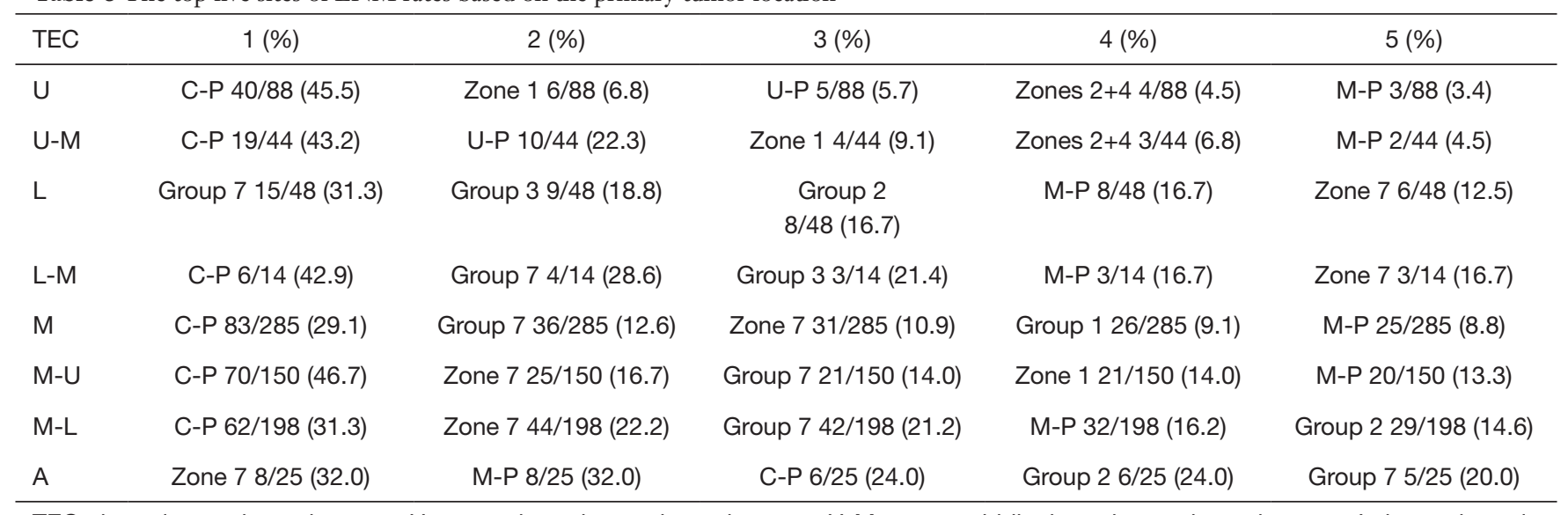

TEC, thoracic esophageal cancer; U, upper thoracic esophageal cancer; U-M, upper-middle thoracic esophageal cancer; L, lower thoracic esophageal cancer; L-M, lower-middle thoracic esophageal cancer; M, middle thoracic esophageal cancer; M-U, middle-upper thoracic esophageal cancer; M-L, middle-lower thoracic esophageal cancer; A, all thoracic esophageal cancer; C-P, cervical paraesophageal; U-P, upper paraesophageal; M-P, middle paraesophageal.

Locoregional recurrence is the main cause for treatment failure after radical esophagectomy, and regional lymph nodes are the most common sites for recurrence (4). Some studies have LNM to be an important prognostic factor for the long-term survival of patients with EC after radical resection (5-7).

Radiotherapy, chemotherapy and surgery are the main components of multidisciplinary treatment of EC. Radiotherapy can improve local control and long-term survival rate significantly. Cheng et al. retrospectively analyzed 1,715 patients with TEC who underwent radical surgery. The 5 -year overall survival rates of surgery and surgery plus postoperative radiotherapy were $21.3 \%$ and $34.2 \%$, respectively. The median survival time was 21.9 and 35.4 months, respectively (8). A study based on the SEER database showed that multimodal therapy (preoperative or postoperative radiotherapy plus surgery) had a better prognosis than surgery only in the 3,292 patients with EC included in the study (9).

The LNM pattern of EC is one of the decisive factors in planning the radiotherapy. It's hard to define the CTV margin for radiotherapy in EC without clear understanding of LNM pattern. Due to the complex lymphatic drainage system of the esophagus, the LNM of EC is characterized by skipped and bidirectional spread (10). It has been systematically elucidated in the previous studies that the depth of tumor invasion, vascular invasion, tumor length, histological type and tumor differentiation affected the LNM pattern of EC, especially the primary tumor location (3,11-13).

A study of 1,077 patients with EC indicated that the LNM was related to the location of the tumor (13). Another study by Cheng et al. involving 1,893 TEC patients from Shandong Cancer Hospital also found that the LNM distribution pattern was primarily dependent upon the location of the primary tumor (14). A meta-analysis of 18,415 patients enrolled in 45 studies by Ding et al. showed the LNM rates in upper, middle and lower TEC were $30.7 \%, 16.8 \%$ and $11.0 \%$ for cervical lymph nodes, $42.0 \%$, $21.1 \%$ and $10.5 \%$ for upper mediastinal lymph nodes, $12.9 \%, 28.1 \%$ and $19.6 \%$ for middle mediastinal lymph nodes, $2.6 \%, 7.8 \%$ and $23.0 \%$ for lower mediastinal lymph nodes, and $9 \%, 21.4 \%$ and $39.9 \%$ for abdominal lymph nodes, respectively (15).

Radiation oncologists always design the CTV according to the location of primary tumor and its lymphatic drainage. Theoretically, the LNM of upper TEC tend to occur in upper mediastinum and neck. The LNM of middle TEC are skipped and bidirectional, so the upper, middle mediastinal and the abdominal lymph nodes all have high chances of metastasis. The lower TEC is prone to metastasize to abdominal lymph nodes. Irrespective of the segment of the esophagus involved, the paraesophageal lymph nodes always have high incidence of metastasis (16).

However, many patients in clinical practice have transsegmental TEC. And there are very limited studies focusing on the difference in characteristics of LNM between transsegmental and mono-segmental TEC. The present study 
was designed to address this issue and provide reference for clinical use while planning radiotherapy.

The U TEC had a higher incidence of LNM in upper mediastinum than U-M TEC (25\% vs. $5.7 \%)$. The most common lymph node regions (LNR) to develop metastases for $\mathrm{U}$ and $\mathrm{U}-\mathrm{M}$ TEC were cervical, upper, middle paraesophageal and zones 1, 2, 4. NCCN guidelines recommend that the superior border of the CTV for $\mathrm{U}$ TEC should include zone 1 and cervical paraesophageal LNR and the inferior border to be extended up to the zone 4 LNR. So, for the U-M TEC, the zone 1, 2, 4 and 5 , cervical, upper and middle paraesophageal LNR were recommended, the same to the U TEC.

Both the L and L-M TEC had high LNM rates in middle mediastinal and abdominal lymph nodes. Additionally, the L-M TEC was prone to metastasize to cervical LNR. The most common sites of LNM rates in L TEC were group 2, 3 and 7, middle paraesophageal, and zone 7. The cases of L-M TEC were limited in this study $(n=14)$. The rates of LNM were $42.9 \%$ (cervical paraesophageal), 28.6\% (group 7), and $21.4 \%$ (group 3), $16.7 \%$ (middle paraesophageal), and $16.7 \%$ (zone 7 ), respectively. It is recommended to include the middle mediastinal and abdominal LNR while delineating the CTV for L and L-M TEC. However, it may be extended up to zone 4 and down to group 7 LNR according to the results of this study. Moreover, unlike in $\mathrm{L}$ TEC, the cervical paraesophageal LNR should be covered in the CTV delineation of L-M TEC as per the findings of this study.

The M, M-U, M-L and A TEC were all characterized by high LNM rates in middle mediastinum with the LNM rates of $18.9 \%, 28.0 \%, 34.3 \%$, and $44.0 \%$, respectively. In the M-U TEC, the most common LNM site was the cervical nodes. LNM tended to appear in the upper mediastinum and neck in $\mathrm{M}-\mathrm{U}$ and $\mathrm{A}$ TEC respectively. In M-L and A TEC, the common LNM site was the abdominal nodes. We also found that the A TEC had high incidence of LNM in all the LNR. Cervical paraesophageal lymph node region is traditionally not added to CTV for $M$ TEC in clinical practice. However, the present study found the LNM rates in neck to be as high as $24.0-46.7 \%$, even for the tumor extending to the lower third of thoracic esophagus. Hence, according to our study, the irradiation field for M-U TEC should be extended from neck to zone 7, including all the paraesophageal regions, zone 1, 2 and 4 . For M-L TEC, the CTV should cover zone 2, 4, 7, left paracardial, lesser curvature, left gastric lymph nodes and all paraesophageal lymph nodes. For A TEC, the CTV may include zone 2, 4, 7, left paracardial, lesser curvature, left gastric and all paraesophageal lymph nodes.

There are several limitations of this study. First, the sample size in our study was not large enough. Second, only cases of esophageal squamous cell carcinoma was included in this study. Third, we did not analyze the LNM pattern of cervical esophageal carcinoma. Moreover, we did not assess the impact of various other parameters such as depth of invasion, vascular invasion, and chemotherapy on LNM pattern. Future prospective studies are required to validate the findings of this study.

\section{Conclusions}

In general, the LNM pattern of trans-segmental and monosegmental TEC are different. The irradiation field must be determined by experienced radiation oncologists according to the primary tumor location. Precise delineation of CTV may improve the local control rate and overall survival for patients with EC.

\section{Acknowledgments}

Thank all those who have contributed to the writing of this thesis.

Funding: None.

\section{Footnote}

Conflicts of Interest: All authors have completed the ICMJE uniform disclosure form (available at http://dx.doi. org/10.21037/apm.2020.04.10). The authors have no conflicts of interest to declare.

Ethical Statement: The authors are accountable for all aspects of the work in ensuring that questions related to the accuracy or integrity of any part of the work are appropriately investigated and resolved. This retrospective study was approved by Fujian Cancer Hospital Institutional Review Board. And the Ethical approval number is YKT2020-001-01. All information of patients was anonymized prior to analysis.

Open Access Statement: This is an Open Access article distributed in accordance with the Creative Commons Attribution-NonCommercial-NoDerivs 4.0 International License (CC BY-NC-ND 4.0), which permits the noncommercial replication and distribution of the article with 
the strict proviso that no changes or edits are made and the original work is properly cited (including links to both the formal publication through the relevant DOI and the license). See: https://creativecommons.org/licenses/by-nc-nd/4.0/.

\section{References}

1. Bray F, Ferlay J, Soerjomataram I, et al. Global cancer statistics 2018: GLOBOCAN estimates of incidence and mortality worldwide for 36 cancers in 185 countries. CA Cancer J Clin 2018;68:394-424.

2. Smyth EC, Lagergren J, Fitzgerald RC, et al. Oesophageal cancer. Nat Rev Dis Primers 2017;3:17048.

3. Huang W, Li B, Gong H, et al. Pattern of lymph node metastases and its implication in radiotherapeutic clinical target volume in patients with thoracic esophageal squamous cell carcinoma: A report of 1077 cases. Radiother Oncol 2010;95:229-33.

4. Cai WJ, Xin PL. Pattern of relapse in surgical treated patients with thoracic esophageal squamous cell carcinoma and its possible impact on target delineation for postoperative radiotherapy. Radiother Oncol 2010;96:104-7.

5. Rice TW, Blackstone EH, Rybicki LA, et al. Refining esophageal cancer staging. J Thorac Cardiovasc Surg 2003;125:1103-13.

6. Zhang HL, Chen LQ, Liu RL, et al. The number of lymph node metastases influences survival and International Union Against Cancer tumor-node-metastasis classification for esophageal squamous cell carcinoma. Dis Esophagus 2010;23:53-8.

7. Isono K, Sato H, Nakayama K. Results of a nationwide study on the three-field lymph node dissection of esophageal cancer. Oncology 1991;48:411-20.

8. Chen J, Wu S, Zheng X, et al. Cervical lymph node metastasis classified as regional nodal staging in thoracic esophageal squamous cell carcinoma after radical

Cite this article as: Huang L, Chen C, Lin M, Li J. Understanding the pattern of lymph node metastasis for transsegmental thoracic esophageal cancer to develop precise delineation of clinical target volume for radiotherapy. Ann Palliat Med 2020;9(3):788-794. doi: 10.21037/apm.2020.04.10 esophagectomy and three-field lymph node dissection. BMC Surg 2014;14:110.

9. Yu J, Ouyang W, Li Y, et al. Value of radiotherapy in addition to esophagectomy for stage II and III thoracic esophageal squamous cell carcinoma: Analysis of surveillance, epidemiology, and end results database. Cancer Med 2019;8:21-7.

10. Tachimori Y. Pattern of lymph node metastases of squamous cell esophageal cancer based on the anatomical lymphatic drainage system: efficacy of lymph node dissection according to tumor location. J Thorac Dis 2017;9:S724-S730.

11. Li H, Zhang Y, Cai H, et al. Pattern of lymph node metastases in patients with squamous cell carcinoma of the thoracic esophagus who underwent three-field lymphadenectomy. Eur Surg Res 2007;39:1-6.

12. Shimada H, Nabeya Y, Matsubara H, et al. Prediction of lymph node status in patients with superficial esophageal carcinoma: analysis of 160 surgically resected cancers. Am J Surg 2006;191:250-4.

13. Natsugoe S, Nakashima S, Matsumoto M, et al. Biologic and imaging diagnosis of lymph node metastasis in esophageal carcinoma. J Surg Oncol 2002;81:25-32.

14. Cheng J, Kong L, Huang W, et al. Explore the Radiotherapeutic Clinical Target Volume Delineation for Thoracic Esophageal Squamous Cell Carcinoma from the Pattern of Lymphatic Metastases. J Thorac Oncol 2013;8:359-65.

15. Ding $X$, Zhang J, Li B, et al. A meta-analysis of lymph node metastasis rate for patients with thoracic oesophageal cancer and its implication in delineation of clinical target volume for radiation therapy. Br J Radiol 2012;85:e1110-9.

16. Wang X, Luo Y, Li M, et al. Recurrence pattern of squamous cell carcinoma in the midthoracic esophagus: implications for the clinical target volume design of postoperative radiotherapy. Onco Targets Ther 2016;9:6021-7. 